I thank Mr W. D. Hoppe for technical assistance and the Medical Research Council of Canada for support. R. J. L. PaultoN

Department of Bacteriology,

University of Saskatchewan,

Saskatoon.

Received March 3; revised May 4, 1970.

${ }^{1}$ Chung, K. L., Hawirko, R. Z., and Isaac, P. K., Canad. J. Microbiol., 10, 43 (1964).

\& Powell, E. O., Biometrika, 42, 16 (1955).

8 Paulton, R.J. L., thesis, Univ. London (1968).

- Hale, C. M. F., and Bisset, K. A., J. Gen. Microbiol., 15, 423 (1956),

"Helmstetter, C. E., and Cooper, S., J. Mol. Biol., 31, 507 (1968).

\section{Fragmentation of Binocular Fusion in Stroboscopic Illumination}

RETINAL images made up of equally spaced parallel vertical lines can readily be fused at disparities differing by a multiple of the line spacing, so giving rise to illusions of depth. In steady light, however, a figure of this kind is normally seen as a single surface, even when tilted away from the frontal plane so that the image lines on one retina intersect the corresponding loci to those on the other retina at several different disparities (Fig. I).

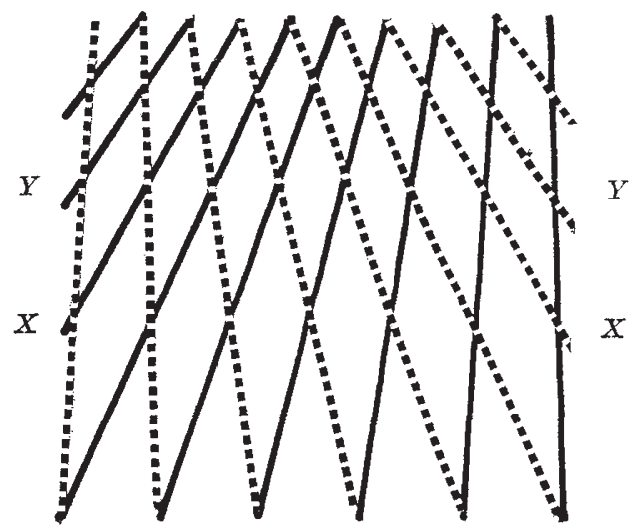
Fig. 1. Left-eye and right-eye views (shown by dotted and solid lines respectively) of part of a receding parallei-line field. When binocularly superposed in stroboscopic light the images can be fused independently

In stroboscopic light at rates of the order of 5 -25 per second, a remarkable fragmentation of the perceived field occurs after some seconds of viewing such a tilted figure. (A line separation of $2 \mathrm{~mm}$ and a viewing distance of 25-100 cm give a good effect.) It breaks up into a series of parallel layers, their position and separation being independent of stroboscopic frequency over the range for which they are visible, but dependent on the distance and angle of inclination of the figure, and of course on the angle of convergence of the eyes. The layers become strikingly visible if the figure is slightly rotated back and forth in its own plane, when the intervening regions seem to move in opposite directions to the layers themselves.

The explanation seems to be that with this intermittent illumination the fusion mechanism regresses to a short range mode of operation in which each local region independently fuses similar portions of the image that happen to lie on corresponding points. In terms of Fig. 1, this means that regions along the lines $X X, Y Y$ and so on should all be seen as lying in the same frontal plane, with the intermediate regions receding from it in nearly parallel layers as observed. As might be expected, the number of layers simultaneously visible (though not their separation) decreases as the flash frequency is increased or decreased from the optimum around $10-20 / \mathrm{s}$; but the upper frequency limit generally increases with practice, and some subjects could train themselves to retain perception of at least two layers even in continuous light.

This phenomenon, which I first noticed some years ago, takes on fresh interest in view of the recent discovery of binocular "disparity detectors" in the visual nervous system ${ }^{1,2}$. It suggests that in studying binocular units the stimulus duration may be a parameter worth examining for its effects on the shape and extent of binocular receptive fields.

I thank the MRC and SRC for financial support.

\section{M. MACKAY}

Department of Communication, University of Keele.

Received April 24, 1970.

${ }^{1}$ Barlow, H. B., Blakemore, C., and Pettigrew, J. D., J. Physiol., 193, 327 (1967).

Nikara, T., Bishop, P. O., and Pettigrew, J. D., Exp. Brain Res., 6, 353 (1968).

\section{Mutants of the Visual Pathway of Drosophila melanogaster}

WiтH the objective of analysing visual processes, we have attempted to generate mutants of Drosophila melanogaster with single-step lesions on the genes controlling the visual pathway ${ }^{1}$. One of these (x-7) had an abnormal electroretinogram (ERG) consisting apparently of responses from the photoreceptor layer alone. Similar ERGs could be obtained from the mutants $\tan ^{1,2}$ and ebony ${ }^{2}$. Indeed, the mutants $x-7$ and tan seemed to be allelic ${ }^{1}$. We now have new mutants, and ERG characteristics suggest genetic blocks in the visual pathway of several of them.

Materials and methods were as described before'. Briefly, males of a wild type $D$. melanogaster stock were treated with ethyl methane sulphonate (EMS) ${ }^{3}$ and mated to virgin females of an attached $X$ chromosome stock (C (1) RM) ${ }^{4}$. Non-phototactic mutants were isolated from the offspring 1 , and their ERG was recorded.

Twenty-three non-phototactic mutants have been isolated so far, and are called $x-1 \ldots x-23$. Mutants $x-1$ to $x-7$ have been described already ${ }^{1}$. The sixteen new mutants were obtained from a total of $30,486 X$ chromosomes tested during a period of about a year. Of these, four are mutants with aberrant ERGs. With the exception of $x-8$ and $x-10$ the external morphology of the new mutants is indistinguishable from wild type. Mutant $\mathrm{x}-8$ is similar in appearance to $\mathrm{x}-1$ (ref. 1 ) and is characterized by forked bristles, fused wing veins and reduced ocelli. Mutant $\mathrm{x}-10$, on the other hand, is similar to the mutant scute (ref. 4) in that some of the scutellar, ocellar and other bristles are missing.

Most of these mutants, including the morphologically distinct $x-8$ and $x-10$, have normal wild type ERGs. But mutants $x \cdot 12,13,14$ and 16 have strikingly aberrant ERGs, shown in Fig. 1 with those of wild type and $x-7$, in the order of progressive departure from the wild type pattern.

The ERG waveform of $\mathrm{x}-14$ (Fig. $1 C$ ) is similar to that of $x-7^{1}$ (Fig. $1 B$ ). They both lack the transients at "on" and "off" and consist only of a sustained, corneal-negative wave, which can be shown to originate from the photoreceptor layer ${ }^{1}$.

The mutants $\mathrm{x}-12, \mathrm{x}-13$ and $\mathrm{x}-16$ have even more abnormal ERGs (Fig. $1 D, E$ and $F$ ). We could obtain no response from x-12. A small response could be obtained from $\mathrm{x}-13$ and a somewhat larger response from $\mathrm{x}-16$, but even the largest response we obtained had an amplitude of only 4-5 $\mathrm{mV}$, while a response of $15 \mathrm{mV}$ amplitude could be routinely obtained from the wild type fly. The waveforms of $x-13$ and $x-16$ ERGs are very similar but 